\title{
The New and Old Issue of "Tissue Morcellation"
}

\author{
Liselotte Mettler ${ }^{*}$, Khulkar Abdusattarova ${ }^{1}$
}

\section{Introduction}

While it is widely accepted that minimal invasive surgical techniques definitely lead to less postoperative pain, decreased morbidity and faster recovery time in comparison to open abdominal procedures the tissue retraction and in our gynecological field myoma and uterine power morcellation in order to avoid larger abdominal incisions or colpotomy has been a challenging field of discussions with many contradictory issues. Morcellation is defined as division and removal in small pieces in the Merriam Webster dictionary. Uterine fibroids are benign neoplasms, affecting $20 \%-40 \%$ of women during their reproductive years (1). When laparoscopic or hysteroscopic operations are performed today electromechanical morcellation is used, which allows removal of uterine fibroids and uterine tissue through port sites. However, morcellation procedures can potentially cause intraoperative dissemination of pathological uterine tissue into the abdominal cavity, especially in unsuspected malignant cases, which may lead to a spreading of the disease and may have negative clinical consequences (2).

The purpose of this editorial is to evaluate advantages and disadvantages of power morcellation and also to determine the incidence of unexpected post-operative histological diagnosis of uterine sarcomas among women who underwent myomectomy and hysterectomy for treatment of benign uterine fibroids.

\section{Historical Background}

Semm and Mettler developed in 1991 the manual morcellator to solve the problem of tissue extraction in Kiel Germany (3). The serrated edged macro-morcellator (SEMM) worked by punching out tissue cylinders of $1,1,5$ and 2 $\mathrm{cm}$ in diameter, in length measuring up to $10-20 \mathrm{~cm} \mathrm{spec-}$ imen particles. Only up 3 times reusable metal serrated edged cylinders were used to cut the tissue, grasped with a big claw forceps. Working only with manual power the technique was time consuming and difficult for the surgeon. SEMM later added battery power and finally electric power to his SEMM, which was produced by WISAP, Germany.

Different types of electro-mechanical morcellators were introduced into the market after 1995. The Steiner mor- cellator was the first one to be Food and Drug Administration (FDA) approved. He used a rotating knife driven by an electric micro engine, controlled via a foot pedal. The cutting cylinder, which was $13 \mathrm{~mm}$ in diameter and $25 \mathrm{~cm}$ long, was placed in a $14 \mathrm{~mm}$ trocar sleeve and protruded a few millimeters past the sleeve of the trocar into the abdomen (4). Carter and Mc-

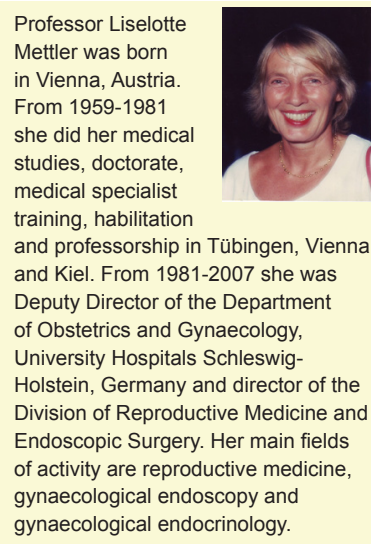
cost analysis of power vs. manual morcellation in 1997. They demonstrated that electromechanical morcellation reduced the average time for extraction of specimen $<100$ $\mathrm{g}$ by 15 minutes and of specimen weighing 401-500 g by 150 minutes. To every bodies understanding this also led to a significant cost reduction despite the more expensive nature of the electromechanical morcellators (5).

\section{Modern Morcellators}

The general engineering of the modern electromechanical morcellators is similar to that described by Steiner. The ideal morcellator is easy to handle, ergonomic, maintains pneumoperitoneum, and enables constant visualization of the rotating knife with minimal operator effort (6).

The morcellator knife was developed in 2000. It was a classic lancet with an interchangeable blade that was inserted through at $10 \mathrm{~mm}$ trocar and used to cut a specimen as it was held between two forceps. A posterior culdotomy was made to remove the small pieces of the specimen. The Sawalhe morcellator, developed by Karl Storz, modified the Steiner model and enabled removal of morcellated tissue from the abdominal cavity via the sleeve, obliviating the need for a posterior culdotomy that was necessary with the morcellator knife (6).

Karl Storz then developed an even more competitive morcellator in 2007 called the Rotocut G1 morcellator. In comparison to the existing Sawalhe model in a study published in 2007, the Rotocut G1 device accomplished sig-

Received 15 November 2015, Accepted 13 December 2015, Available online 22 December 2015

${ }^{1}$ Department of Gynecology and Obstetrics, University Hospitals Schleswig-Holstein, Kiel, Germany.

*Corresponding author: Liselotte Mettler; Email: lmettler@emai.uni-kiel.de 
nificantly shorter morcellation time, operative time and duration of anesthesia. Fewer and longer pieces of tissue due to a more effective power output and drive transmission gave a faster removal time. In this model, the generator is located in the hand piece and is activated by a foot pedal (6).

The Gynecare Morcellex tissue morcellator developed by Ethicon Inc. is another popular power morcellator. Unlike the Rotocut G1, the Gynecare Morcellex does not require a foot pedal. In 2009, a randomized controlled trial was initiated to compare the two popular models, the Gynecare Morcellex and the Rotocut G1. There was no statistical difference between the two groups in regards to operative time, morcellation time, weight of excised pieces, blood loss/blood transfusion, intra or post operative complications, postoperative pain, hospitalization or time to return to full working activity. Only the VAS score ranging from 0 (low handling, easy) to 10 (high handling, difficult) the 2 morcellators were evaluated. There was a significant difference in ease of use, with the Gynecare Morcellex having a higher handling score (average 7.0 for supracervical hysterectomy and 7.2 for myomectomy) (7).

In July 2014 the Gynecare Morcellex was withdrawn from the market by Johnson and Johnson after a statement discouraging the use of power morcellators was released by the FDA (See "Updated FDA Recommendations").

\section{Transcervical Morcellation}

Morcellation has also been used by a transcervical approach after a supracervical hysterectomy. The steps are essentially the same; however, instead of extending an abdominal wall incision by dilation, the cervical canal is dilated until the morcellator cannula can be inserted. We used this technique a lot for morcellation after the LSH procedures. A longer cannula and blade must be used to traverse the vaginal canal. Morcellation is then completed in the same manner and the dilated cervical may be closed using a single stitch laparoscopically. This technique allows the surgeon to avoid making a large abdominal wall incision and thus decreases the risk of future herniation. Details were nicely described in the literature by Rosenblatt et al in 2010 (8).

\section{Complications}

As morcellators are frequently used in laparoscopic and hysteroscopic surgery, morcellator-related injuries have also increased. These injuries range from direct surgical risks resulting in immediate complications to sequelae of morcellated tissue fragments and morcellation of preoperatively undiagnosed malignancy resulting in possibly more long term complications.

Milad and Milad performed a systematic review using a literature search and a search of the FDA medical device reporting (MDR) and Manufacturer and User Facility Device Experience (MAUDE) databases to determine the frequency of immediate morcellator injuries from 1992 to 2012 (9). This study examined morcellation after hysterectomy, myomectomy, nephrectomy, splenectomy, and "others." They found a total of 55 complications, specifically including injuries to the small and large bowels $(n=31)$, vascular system $(n=27)$, kidney $(n=3)$, ureter $(n=3)$, bladder $(n=1)$ and diaphragm $(n=1)$. Most $(66 \%)$ of these injuries were identified intraoperatively. Six patients died of morcellator related complications.

Long-term complications of morcellation include retained or parasitic tissue, leiomyomatosis or dissemination of undiagnosed malignancy. The term "iatrogenic parasitic myoma" has been developed to describe the formation of new myomas that are not attached to the uterus presenting after uterine or myoma morcellation. When fragments of myomas are left behind following morcellation and become implanted in normal tissue in the abdominal cavity uterine myomatosis may occur.

Iatrogenic endometriosis has been reported to occur after uterine morcellation, but not all of the literature is consistent. A case report by Sepilian and Della Badia demonstrated widespread endometriosis and symptoms of cyclic pelvic pain six months following morcellation after a supracervical hysterectomy performed for uterine fibroids with an absence of endometriosis (10).

\section{The Malignancy Potential at Tissue Dissemination}

The biggest concern in regards to dissemination of tissue during morcellation is the inadvertent dissemination of malignancy.

\section{Endometrial Adenocarcinoma}

If morcellation of endometrial adenocarcinoma can cause cancerous tissue to be spread throughout the abdomen and lead to possible upstaging of an existing malignancy this does appear as a maximal thread. Morcellation of endometrial adenocarcinoma can usually be avoided by appropriate pre-operative evaluation with endometrial biopsy or dilation and curettage; however, this is not always accurate. Studies have shown a discrepancy of 10\%-16\% in histologic diagnosis with endometrial biopsy or curettage when compared to hysterectomy. A recent European evaluation showed a concordance rate of only $62 \%$ and $67 \%$ of endometrial biopsy and curettage (respectively) when compared to hysterectomy (11).

\section{Leiomyosarcoma}

Since the beginning of introducing tissue morcellation into gynecology it was strongly advised to exclude any case of unclear preoperative pathology from morcellation. Of greatest concern is the inadvertent morcellation of a leiomyosarcoma as leiomyosarcoma is the most common malignant nonepithelial tumor of the uterus (12) representing $1 \%-2 \%$ of all uterine malignancies. It is an aggressive malignancy with 5 -year survival rates of $18.8 \%$ to $65 \%$. Particularly women in the perimenopausal years are affected. Their median age of 52 years and sarcomas are very rare in women below the age of 40 years.

Mostly this malignancy reveals a rapidly growing uterus; however, because it is uncommon, only $0.23 \%$ of patients with this finding will have leiomyosarcoma Preopera- 
tive diagnosis is challenging because the symptoms and the clinical appearance associated with leiomyosarcoma are nearly identical to benign leiomyoma. Magnetic resonance imaging (MRI) with diffusion-weighted imaging and PET/CT with F-FDG have both been evaluated in the pre-operative diagnosis of leiomyosarcoma $(13,14)$; neither has been sufficiently proven to offer accurate diagnosis. Utilizing serum lactate dehydrogenase (LDH) and its isoenzymes, especially isozyme type 3 , has also been utilized to distinguish leiomyosarcoma from benign leiomyoma and is a promising technique. The correlation was first seen in a series of 1886 patients who underwent hysterectomy for uterine myomas, 7 of which were found to have leiomyosarcoma. Three of these 7 patients had an elevated LDH and these 3 patients had tumors with worse prognostic factors. In a follow up study evaluating $10 \mathrm{pa}-$ tients with leiomyosarcoma and 130 patients with degenerated leiomyoma, all patients with leiomyosarcoma had elevated total LDH and LDH isozyme type 3. When LDH was combined with MRI, the specificity, positive predictive value and negative predictive value were all $100 \%$ in this particular study (15). MRI and LDH should be performed in all slightly unclear preoperative evaluations if power morcellation stands up for discussion

There are currently 9 studies in the literature on unsuspected leiomyosarcomas in patients who had a hysterectomy or myomectomy for presumed benign disease (16). The rate of leiomyosarcoma in these studies ranges from $0 \%-0.49 \%$ with the average being $0.18 \%$. The FDA recently released a statement quoting the incidence to be 1 per 350 or $0.29 \%$ (17). This incidence has been challenged by Elizabeth Pritts et al at the FDA meeting. Her more extensive evaluation of the literature was correlated to a much lower risk of leiomyosarcoma. Morcellating these unsuspected malignancies can result in upstaging and a worse prognosis $(18,19)$.

Another retrospective study of 58 patients, 19 of whom underwent morcellation, showed a significant increase in the risk of abdominal/pelvic recurrences and shorter median recurrence-free survival in patients with unsuspected leiomyosarcomas who underwent intraperitoneal morcellation (20).

Due to these controversial findings and reports, the FDA put out a statement discouraging the use of power morcellators, citing safety concerns, mostly the inadvertent dissemination for occult uterine cancer in patients undergoing hysterectomy and myomectomy for presumed leiomyomata (17). They quote other options to intracorporeal morcellation including removing the uterus through a mini-laparotomy or morcellating the uterus inside a laparoscopic bag (21). The AAGL states that when comparing the risks involved in open hysterectomy versus those of power morcellation, gynecologists should improve but not abandon power morcellation, and that power morcellation with appropriate informed consent should remain available to appropriately screened, low risk women (22). Also Pritts et al (23) talk of the necessity of increasing our awareness towards malignancies prior to scheduling a morcellation of tissue, but describe a much lower risk than 1 sarcoma in 1000 morcellations of fibroid tissue.

\section{Contained Morcellation - Does It Diminish the Risk?}

Fibroid tissue morcellation within a bag is also called contained morcellation. Cohen et al came out with a feasibility study in September 2014 reporting 73 successful cases of morcellation of uteri or myomas with and insufflated bag. There were no complications in this report and no visual evidence of tissue dissemination outside of the isolation bag. The bag used in this case was developed by one of the authors specifically for this use (24).

Recently many different forms of theses bags are being evaluated. We work on a technique homogenizing the tissue in a bag to powder, to be extracted by a catheter technique to be later evaluated for malignancy by genetic technology.

Despite all these reports on the danger of spreading malignant disease at morcellation, many of these data are still limited and controversial. In a systematic review of 6 studies, data seemed to be highly biased and of poor quality, resulting in the author's conclusion that there is no reliable evidence that morcellation significantly results in tumor upstaging or in poorer patient outcome. There is also no evidence from these studies that power morcellation affects patient outcomes differently than any other type of morcellation, or even simple myomectomy. Already a myoma enucleation, the opening of the pseudo capsule in a case of an adenomatoid tumour or a sarcoma may have the same risk potential than a careful morcellation.

\section{Morcellation Evaluation at the University Department Obstetrics \& Gynecology in Kiel, Germany Methods}

We included into our retrospective "morcellation evaluation study" patient's data from 2010 to 2015. Proportion confidence intervals were calculated using the Wilson method. Continuous variable confidence intervals were calculated using Student's t-distribution. The retrospective preliminary results are discussed as follows.

\section{Results}

Out of a total of 819 patients who were operated with myomectomies or hysterectomies for uterine fibroids 612 were operated by laparoscopy. Power morcellation was only applied in 457 cases $=55.8 \%$. The mean age of all patients with uterine fibroids was $44.45 \pm 9.19$. Uterus-preserving surgeries were more frequently performed in younger patients. The mean age of laparoscopic myomectomy patients was $36.73 \pm 6.54$, in abdominal myomectomy $35 \pm 4.7$, and in hysteroscopic myomectomy $43.62 \pm 10.27$ years.

The mean cumulative diameters of uterine fibroids were significantly higher in laparoscopic operations where the morcellator was used: total laparoscopic hysterectomy (TLH) $11.2 \pm 2.95 \mathrm{~cm}$; laparoscopic supracervical hysterectomy (LSH) $8.92 \pm 3.53 \mathrm{~cm}$; laparoscopic myomectomy $10.08 \pm 4.02 \mathrm{~cm}$ than in procedures without 
morcellation TLH $5.87 \pm 2.13$; laparoscopic myomectomy $4.65 \pm 1.04 \mathrm{~cm}$.

In laparoscopic hysterectomies, where power morcellator was used the mean uterine weights were approximately two fold higher in TLH $(491.46 \pm 227.41 \mathrm{~g})$ and LSH $(326.12 \pm 268.01 \mathrm{~g})$ in comparison to TLH without morcellation $(164.25 \pm 56.94 \mathrm{~g})$. The mean uterus weights in vaginal hysterectomies were $160.39 \pm 51.09 \mathrm{~g}$, which were approximately the same in TLH operations (164.25 \pm 56.94 g) without morcellation.

The mean weights of fibroids in laparoscopic myomectomies with morcellation were $90.16 \pm 104.78 \mathrm{~g}$, whereas without morcellation the weights were only $19.42 \pm 6.07 \mathrm{~g}$. In hysteroscopic myomectomies the mean weights of fibroids were $12.81 \pm 6.44 \mathrm{~g}$. The mean weight of fibroids in open abdominal myomectomies were $481.38 \pm 333.05 \mathrm{~g}$.

\section{Discussion}

All these 819 patients who were operated for uterine fibroids, 612 had laparoscopic procedures but only in 457 cases (55.8\%) laparoscopic morcellation techniques were used. Within these 5 years only in 1 patient with presumed uterine fibroids, who underwent LSH in histopathology an unsuspected endometrial stromal sarcoma was detected; this patient was 54 years old and consequently follow-up with surgeryaccording to the oncologic guidelines for uterine sarcomas.

\section{Conclusions}

Laparoscopic power morcellation for tissue extraction should be performed only in cases where any malignancy potential can be most likely excluded. All patients who undergo laparoscopic or hysteroscopic surgery for myoma enucleation or hysterectomy and face morcellation during their surgery should be informed about the possible risks of morcellation in cases of difficult to diagnose and rare cases of unexpected malignancies. Special attention is advised in patients over the age of 50 for morcellation, as most of observed preoperatively not suspected rare sarcoma cases were in females beyond the age of 50 years.

The final advice concerning myomectomy alone "power-morcellation," "contained morcellation" or "open surgery" to avoid unprotected morcellation is still outstanding. Who knows if even myomectomy at open or vaginal surgery does not carry the same risks as we are discussing.

\section{Ethical Issues}

Not applicable.

\section{Conflict of Interests}

The authors have no conflicts of interest to disclose.

\section{References}

1. Müller A, Thiel F, Binder $\mathrm{H}$ et al. Myome-Teil 1. Geburtshilfe Frauenheilk. 2004;64(10):R229-R244. doi:10.1055/s-2004-830411.

2. Beckmann MW, Juhasz-Böss I, Denschlag D, et al. Surgical Methods for the Treatment of Uterine
Fibroids - Risk of Uterine Sarcoma and Problemsof Morcellation: Position Paper of the DGGG. Geburtshilfe Frauenheilkd. 2015;75(2):148-164.

3. Semm K. Morcellement and suturing using pelviscopy: not a problem any more (Article in German). Geburtshilfe Frauenheilkd. 1991;51(10):843-846.

4. Steiner RA, Wight E, Tadir Y, Haller U. Electrical cutting device for laparoscopic removal of tissue from the abdominal cavity. Obstet Gynecol. 1993;81(3):471-474.

5. Carter JE, McCarus SD. Laparoscopic myomectomy. Time and cost analysis of power vs. manual morcellation. J Reprod Med 1997;42:383-388.

6. Brucker S, Solomayer E, Zubke W, et al. A newly developed morcellator creates a new dimension in minimally invasive surgery. J Minim Invasive Gynecol. 2007;14:233-239.

7. Zullo F, Falbo A, Iuliano A, et al. Randomized controlled study comparing the Gynecare Morcellex and Rotocut G1 tissue morcellators. J Minim Invasive Gynecol. 2010;17(2):192-199. doi:10.1016/j. jmig.2009.11.009.

8. Rosenblatt PL, Makai G, DiSciullo AJ. Laparoscopic supracervical hysterectomy with transcervicalmorcellation. J Minim Invasive Gynecol. 2010;17(3):331-336. doi:10.1016/j.jmig.2010.02.004.

9. Milad MP, Milad EA. Laparoscopic morcellatorrelated complications. J Minim Invasive Gynecol 2014;21:486-491.

10. Sepilian V, Della Badia C. Iatrogenic endometriosis caused by uterine morcellation during a supracervical hysterectomy. Obstet Gynecol. 2003;102:1125-1127.

11. Gungorduk K, Asicioglu O, Ertas IE, et al. Comparison of the histopathological diagnoses of preoperative dilation and curettage and Pipelle biopsy. Eur J Gynaecol Oncol. 2014;35(5):539-543.

12. Della Badia C, Karini H. Endometrial stromal sarcoma diagnosed after uterine morcellation in laparoscopic supracervical hysterectomy. J Minim Invasive Gynecol 2010;17:791-793.

13. Sato K, Yuasa N, Fujita M, et al. Clinical application of diffusion-weighted imaging for preoperative differentiation between uterine leiomyoma and leiomyosarcoma. Am J Obstet Gynecol. 2014;210(4):368.e1-8. doi:10.1016/j.ajog.2013.12.028.

14. Seki K, Hoshihara T, Nagata I. Leiomyosarcoma of the uterus: ultrasonography and serum lactate dehydrogenase level. Gynecol Obstet Invest. 1992;33:114-118.

15. Goto A, Takeuchi S, Sugimura K, Maruo T. Usefulness of Gd-DTPA contrast-enhanced dynamic MRI and serus determination of $\mathrm{LDH}$ and its isozymes in the differential diagnosis of leiomyosarcoma from degenerated leiomyoma of the uterus. Int J Gynecol Cancer. 2002;12(4)354-361.

16. Stine JE, Clarke-Pearson DL, Gehrig PA. Uterine morcellation at the time of hysterectomy: techniques, risks, and recommendations. Obstet and Gynecol 
Surv. 2014;69(7): 415-425.

17. US Food and Drug Administration (FDA). Laparoscopic Uterine Power Morcellation in Hysterectomy and Myomectomy: FDA Safety Communication. http://www.fda.gov/ medicaldevices/safety/alertsandnotices/ucm393576. htm. Published 2014 November.

18. Park JY, Park SK, Kim DY, et al. The impact of tumor morcellation during surgery on the prognosis of patients with apparently early uterine leiomyosarcoma.Gynecol Oncol. 2011;122:255-259.

19. Einstein MH, Barakat RR, Chi DS, et al. Management of uterine malignancy found incidentally after supracervical hysterectomy or uterine morcellation for presumed benign disease. Int J Gynecol Cancer 2008;18:1065-1070.

20. George S, Barysauskas C, Serrano C, et al. Retrospective cohort study evaluating the impact of intraperitoneal morcellation on outcomes of localized uterine leiomyosarcoma. Cancer. 2014;120(20):31543158. doi:10.1002/cncr.28844.

21. SGO Position Statement: Morcellation. SGO website. https://www.sgo.org/newsroom/position-statements2/morcellation. Accessed October 2014. Published December 2013.

22. Brown, Jubilee and board of trustees. AAGL Statement to the FDA on Power Morcellation. http://www.aagl. org/. Published July 2014.

23. Pritts EA, Parker WH, Brown J, Ollive DL. Outcome of occult uterine leiomyosarcomaafter surgery for presumed uterinefibroids: A systematic review. J Minim Invasive Gynecol. 2015;22(1):26-33. doi:10.1016/j.jmig.2014.08.781.

24. Cohen SL, Einarsson JI, Wang KC, et al. Contained power morcellation within an insufflated isolation bag. Obstet Gynecol. 2014;124(3):491-497. doi:10.1097/AOG.0000000000000421.

Copyright (C) 2016 The Author(s); This is an open-access article distributed under the terms of the Creative Commons Attribution License (http://creativecommons.org/licenses/by/4.0), which permits unrestricted use, distribution, and reproduction in any medium, provided the original work is properly cited. 